\title{
Reply to Emv2, the only endogenous ecotropic murine leukemia virus of $\mathrm{C} 57 \mathrm{BL} / 6 \mathrm{~J}$ mice
}

\author{
Kang-Hoon Lee and Kiho Cho*
}

\section{Abstract}

This correspondence was written in response to the comments by Young et al. Following careful evaluation of the relevant dataset, each of the points brought up by Young et al. has been addressed in this response. We anticipate this will clarify our findings regarding $E_{\text {m }} V_{\text {men, }}$ an ecotropic endogenous retrovirus that was shown to have cerebellum-specific and age-dependent expression patterns in C57BL/6J mice.

\section{Correspondence}

The authors of the paper by Lee et al [1]. appreciate the interest shown and comments by Young et al [2].

Young et al. contend that Emv2 is the only endogenous ecotropic murine leukemia virus in C57BL/6J mice, although not annotated on the current NCBI reference genome assembly, Build 37.2, and maps to the distal region of chromosome 8 based on previous genetic studies.

As stated in the paper by Lee et al., the Emv2 locus was annotated in the NCBI database Build 37.1 when analyzed around November 2010. At that time, the annotated Emv2 locus did not contain any sequences similar to mouse endogenous retroviruses. Interestingly, a survey of the latest NCBI database (Build 37.2) for the Emv2 locus on December 08, 2011, in response to the correspondence by Young et al., yielded no annotated information about the Emv2 locus, and it appears that the previous annotations may have been removed. During the course of our analysis regarding the Emv2 locus in the $\mathrm{C} 57 \mathrm{BL} / 6 \mathrm{~J}$ mouse genome, screenshots of the data were captured and recorded, and revealed the annotation of the Emv2 locus on chromosome 8, which is distantly located from the ERV $V_{\text {mch } 8}$ locus (Figure 1). Although the paper by Lee et al. uses Build 37.1 to show the annotation for Emv2, this annotation for Emv2 appears as far back as 2006 (NCBI, when reverting to the previous build version 36.1).

This was cited in the paper by Lee et al.:

\footnotetext{
* Correspondence: kcho@ucdavis.edu

Departments of Surgery, University of California, Davis and Shriners Hospitals for Children Northern California, Sacramento, CA 95817, USA
}

In addition, Emv2 is located/annotated at $67.0 \mathrm{cM}$, $\sim 11.4 \mathrm{cM}$ upstream of the $E R V_{\text {mch }}$ locus ( 78.4 cM), according to a survey of the NCBI map viewer (http://www.ncbi.nlm.nih.gov/projects/mapview)... Unexpectedly, we were unable to retrieve the nucleotide sequence, which is presumed to be the Emv2 provirus, from the Emv2 locus annotated in the ... However, it is still a possibility that ERV $V_{\text {mchs }}$ shares the same locus on chromosome 8-qE1 region with Emv2 with an assumption that the NCBI annotation information regarding the Emv2 locus needs to be revised.

As indicated in the paper by Lee et al., we were not able to find the "full/complete" sequence information from the NCBI database using the keyword "Emv2", and it was unclear why there was no "full/complete" sequence information deposited into the database when substantial work in regard to Emv2 has been reported. When preparing the manuscript, a thorough survey of relevant scientific publications for Emv2 also yielded neither "full/ complete" nor partial sequence information.

We suggest a further study is needed to confirm whether the ERV $V_{\text {mch8 }}$ locus indeed matches the Emv2 locus. Importantly, the difference in distance between the annotated Emv2 locus (chromosome 8 at $\sim 67 \mathrm{cM}$ region) and the ERV $V_{\text {mch8 }}$ locus (chromosome 8 at $\sim 78.4 \mathrm{cM}$ region) needs to be clarified (Figure 1). In addition, it appears that the result from Young et al.'s "in silico Southern blot" analysis referencing the $E R V_{\text {mch8 }}$ sequence may not be sufficient to confirm that $\mathrm{ERV}_{\text {mch8 }}$ is Emv2. A wellfocused experiment is needed to determine whether the previously reported Emv2 locus matches to the $\mathrm{ERV}_{\mathrm{mch}} 8$ 


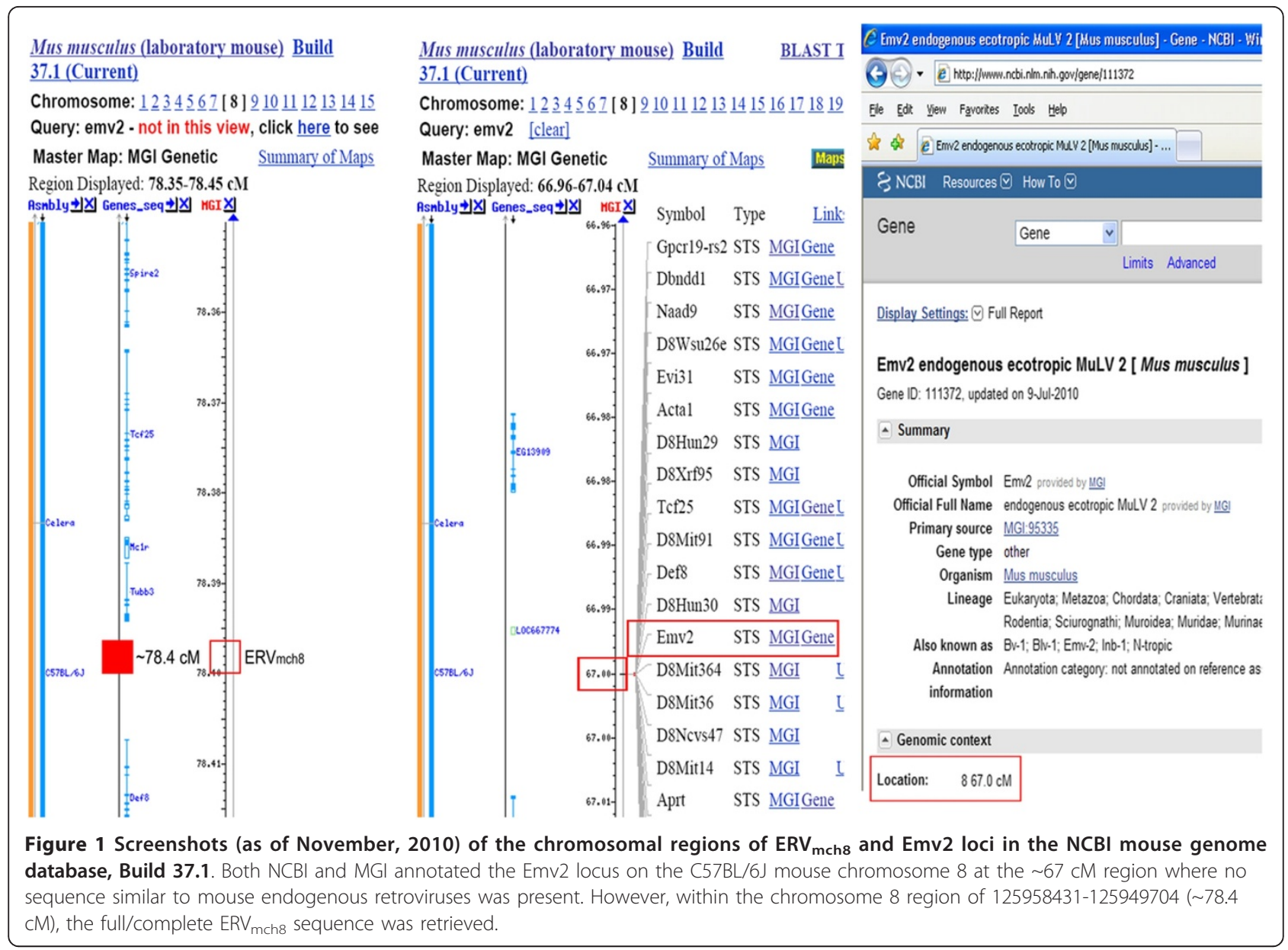

locus. For example, to help determine whether ERV $\mathrm{mch}_{\text {m } 8}$ is indeed Emv2, a set of Southern blot analyses can be performed using the genomic DNAs from various mouse strains, including the mouse strains reported in the studies referenced by Young et al., with probes designed from the "full/complete" ERV $\mathrm{mch}_{\text {8 }}$ sequence, in addition to the $\mathrm{pEco}$ probe. It will be very helpful if the original DNAs, which were used for the Emv studies referenced by Young et al., are still available. In addition, it may be necessary to clone and sequence the $\mathrm{ERV}_{\text {mch8}} / \mathrm{Emv} 2$ locus in these mouse strains to examine whether there are any sequence polymorphisms among the presumed loci in different mouse genomes.

Young et al. contend that the terminology "novel ecotropic provirus" and labeling the virus as having an "intact coding potential" is misleading. Throughout the manuscript, the word "novel" was used only once for the endogenous retrovirus $\left(E R V_{\text {mch8 }}\right)$ in the title of Figure 1, primarily because, as previously stated, the NCBI Emv2 annotation locus was completely different from the ERV $\mathrm{mch}_{\text {m }}$ locus (Please refer to Figure 1 of this response.) Furthermore, the title of the manuscript does not contain the word "novel." In fact, the paper suggests that the NCBI annotation of Emv2 may need revision. Additionally, the phrase "intact coding potential" was used to indicate an "intact open reading frame" is present, without suggesting a precise function of the protein to be coded. The abstract of the paper by Lee et al. explains:

It appears that ERV $V_{\text {mchs }}$ shares the same genomic locus with a replication-incompetent MuLV-ERV, called Emv2; however, it was not confirmed due to a lack of relevant annotation and Emv2 sequence information.

Young et al. also advise that different reference virus choices should be made for phylogenetic classification. We appreciate Young et al.'s suggestion in regard to the choice of reference sequences; however, our analyses focused on the evaluation of relatedness at the nucleotide level, not at the virus level.

Additionally, Young et al. remind us that previous studies by Jenkins et al., using a Southern hybridization 
method, found Emv2 only on C57BL/6J and a few related mouse strains. Young et al. also suggest that the PCR approach employed by Lee et al. uses "primers that are wrongly presumed specific for the 'ER $V_{\text {mchs }}$ ' sequence, [and] found an ecotropic provirus on chromosome 8 in a wide variety of strains."

The primer set used in the paper by Lee et al. was designed to amplify a unique region from the end of the env gene to the middle of the LTR of ERV $\mathrm{mch}_{\text {m } 8}$ in the C57BL/6J mouse genome. In silico PCR (UCSC) retrieved only one locus for $\mathrm{ERV}_{\text {mch8}}$, which is located on chromosome 8 of the C57BL/6J mouse genome and the only copy of an ecotropic MuLV found in that genome. The data presented in the paper by Lee et al. intend to only show the general distribution of the 'ERV $\mathrm{m}_{\mathrm{mch}}$ sequence' throughout the genomes of 57 mouse strains and do not specifically refer to an $E R V_{\text {mch8 }}$ sequence on chromosome 8 (since the copy number or locations can vary depending on the strain). As stated in the results section of the paper by Lee et al., the variability of size and intensity of these bands suggests "polymorphisms in the sequences and/or copy numbers."

It should also be noted that both the Southern blot and PCR protocols share a specific step which is "nucleic acid" to "nucleic acid" hybridization. As such, both protocols harbor the potential for false identification of the intended target DNAs. Our laboratory also routinely includes relevant RT-negative controls for RT-PCR analyses.

We hope that the responses by Lee et al. clarify the comments brought up by Young et al. We anticipate this correspondence will clarify our findings regarding $E R V_{\text {mch8 }}$, an ecotropic endogenous retrovirus that was shown to have cerebellum-specific and age-dependent expression patterns in C57BL/6J mice.

\section{Acknowledgements}

We would like to acknowledge Tajia Green and Debora Lim for editorial assistance.

Authors' contributions

The authors contributed equally to this article.

\section{Competing interests}

The authors declare that they have no competing interests.

Received: 7 February 2012 Accepted: 22 March 2012

Published: 22 March 2012

\section{References}

1. Lee KH, Horiuchi M, Itoh T, Greenhalgh DG, Cho K: Cerebellum-specific and age-dependent expression of an endogenous retrovirus with intact coding potential. Retrovirology 2011, 8:82.

2. Young GR, Kassiotis G, Stoye JP: Emv2, the only endogenous ecotropic murine leukemia virus of C57BL/6J mice. Retrovirology 2012, 9:23. doi:10.1186/1742-4690-9-24

Cite this article as: Lee and Cho: Reply to Emv2, the only endogenous ecotropic murine leukemia virus of C57BL/6J mice. Retrovirology 2012 9:24.

\section{Submit your next manuscript to BioMed Central} and take full advantage of:

- Convenient online submission

- Thorough peer review

- No space constraints or color figure charges

- Immediate publication on acceptance

- Inclusion in PubMed, CAS, Scopus and Google Scholar

- Research which is freely available for redistribution

Submit your manuscript at www.biomedcentral.com/submit 